\title{
Bibliographie exhaustive de Jerzy Kalinowski
}

\section{Tadeusz Kwiatkowski et Agnès Bastit}

\section{(2) OpenEdition}

\section{Journals}

Édition électronique

URL : http://journals.openedition.org/philosophiascientiae/507

DOI : 10.4000/philosophiascientiae.507

ISSN : 1775-4283

Éditeur

Éditions Kimé

Édition imprimée

Date de publication : 1 avril 2006

Pagination : 195-221

ISBN : 2-84174-392-6

ISSN : $1281-2463$

\section{Référence électronique}

Tadeusz Kwiatkowski et Agnès Bastit, «Bibliographie exhaustive de Jerzy Kalinowski », Philosophia Scientiæ [En ligne], 10-1 | 2006, mis en ligne le 10 juin 2011, consulté le 16 janvier 2021. URL : http:// journals.openedition.org/philosophiascientiae/507 ; DOI : https://doi.org/10.4000/ philosophiascientiae.507 


\section{Bibliographie exhaustive de Jerzy Kalinowski}

Tadeusz Kwiatkowski

Université de Lublin

Agnès Bastit

Université de Metz

\section{Introduction}

La présente bibliographie a été constituée sur la base de celle parue en 1995-1996, sous le titre de "Bibliografia podmiotowa i przedmiotowa Profesora Jerzego Kalinowskiego", Summarium 24-25 (44-45), Lublin, 1995-1996, elle-même inspirée, en ce qui concerne les années antérieures à 1983, de la bibliographie établie par R. Bozzi, La logique deontica di Georges Kalinowski, Naples, 1984, 193-204. Nous l'avons complétée par les quelques titres postérieurs à 1995 .

Nous remercions Madame Florence Bouchet (Centre Commun de Documentation en Sciences Humaines et Sociales, Université Nancy 2) de son assistance lors du travail de vérification des entrées en langue espagnole. 


\section{Principales abréviations :}

ATK Akademia Teologii Katolickiej.

IRETIJ Institut de Recherche et d'Études pour le Traitement de l'Information Juridique.

L.G.D.J. Librairie Générale de Droit et de Jurisprudence.

KUL Katolicki Uniwersytet Lubelski.

TFP Towarzystwo Filozofii Praktycznej.

TNKUL Towarzystwo Naukowe Katolickiego Uniwersytetu Lubelskiego.

UMCS Uniwersytet Marie-Curie-Sktodowskiej.

\section{- Les livres de Jerzy Kalinowski}

1996 La logique déductive. Essai de présentation aux juristes, Paris : PUF, 1996, (172 pages).

1992 Expérience et phénoménologie : Husserl, Ingarden, Scheler, Paris : Editions Universitaires, 1992.

1991 La phénoménologie de l'homme chez Husserl, Ingarden et Scheler, Paris : Editions Universitaires, 1991.

1987 Autour de personne et acte de Karol Cardinal Wojtyla. Une rencontre de la phénoménologie et du thomisme, Aix-en-Provence : Presses Universitaires d'Aix-Marseille, 1987.

(recueil d'articles déjà parus, complété par un avant-propos et par deux annexes originales).

1985 Sémiotique et Philosophie, Paris : Editions Hadès Benjamins, 1985.

1982 Concepto, fundamento y concreción del derecho, Buenos Aires : Abeledo-Perrot, 1982.

(traduction espagnole des articles suivants : [Kalinowski 1963a] : Lex et ius. A propos d'une définition du droit de Michel Villey, Archives de Philosophie du Droit, 8, 285-291, 1963, [Kalinowski 1964a] : Logique et philosophie du droit subjectif, Archives de Philosophie du Droit, 9, 37-43, 1964, [Kalinowski 1966c] : La pluralité ontique en philosophie du droit, Revue philosophique de Louvain, 64, 263-280, 1966, [Kalinowski 1973d] : Le fondement objectif du droit d'après la Somme de Théologie de saint Thomas d'Aquin, Archives de Phi- 
losophie du Droit, 18, 59-75, 1973, [Kalinowski 1969f] : Note sur le rapport entre le fait et le droit, Rivista internazionale di filosofia del diritto, 46, 411-422, 1969, [Kalinowski 1960a] : Le droit naturel et la personne humaine, Chronique Sociale de France, 68, 195-199, 1960, [Kalinowski 1972c] : Philosophie et logique de l'interprétation en droit, Archives de Philosophie du Droit, 17, 39-49, 1972 et [Kalinowski 1967b] : Application du droit et prudence, Archiv für Rechts-und Sozialphilosophie, 53, 161-178, 1967.).

1981 L'impossible métaphysique (avec en annexe trois lettres inédites d'E. Gilson), Paris : Beauchesne, 1981.

Traduction italienne, par G. Ferrari, L'impossibile metafisica, Genova : Marietti, 1991.

1978 Lógica de las normas y lógica deóntica. Posibilidad y relaciones, Numéro spécial des Cuadernos de Metodología y filosofía del derecho, 1, Valencia : Universidad de Carabobo, (Venezuela), 1978.

(version castillanne par R. Carrion Wam, précédée d'un avant-propos original, des trois articles suivants : [Kalinowski 1977a] : Über die Bedeutung der Deontik für Ethik und Rechtsphilosophie, Deontische Logik und Semantik, hrsg. A. C. Conte, R. Hilpinen, G. H. von Wright, Wiesbaden (Athenaion), 101-130, 1977, [Kalinowski 1975f] : Du métalangage en logique. Réflexions sur la logique déontique et son rapport avec la logique des normes, Documents de travail et prépublications, Urbino (Université d'Urbino), Centre International de Sémiotique et de Linguistique, A (48), 1-28, 1975 et [Kalinowski 1976f] : O poslugiwaniu sie w logice deontycznej jezykami preskryptywnym i deskryptywnym. Dwa pytania na temat "derived obligation", Studia Filozoficzne, 3, nr. specjalny w 90 rocznice urodzin Profesora Tadeusza Kotarbinskiego, 121-128, 1976.).

1972a Etudes de logique déontique, I (1953-1969), Préface de R. Blanché, Paris : L. G. D. J., 1972.

1972b La logique des normes, Paris : PUF, 1972.

Traduction allemande (par Wolfgang Klein) : Einführung in die Normenlogik, Frankfurt-am-Main : Atheneum, 1972.

Traduction espagnole (par J. R. Cappela) : Lógica del discurso normativo, Madrid : Ed. Tecnos, 1975.

Traduction polonaise : Logika norm, Lublin : Daimonion, 1993.

1969 Querelle de la science normative (Préface de Michel Willey), Paris : L. G. D. J., 1969. 
(Traduction italienne : Disputa sulla scienza normativa, a cura di G. F. Ferrari, Padova : CEDAM, 1982.

1967 Le problème de la vérité en morale et en droit, Lyon : Ed. Vitte, 1967.

Traduction espagnole (par E. Mari) : Et problema de la verdad en la moral y en el derecho, Buenos Aires : Editorial Universitaria, 1979.

1966 Initiation à la philosophie morale (Préface de J. Folliet), Paris : Société d'Editions Internationales, 1966.

1965 Introduction à la logique juridique, (Préface de Ch. Perelman), Paris : L.G.D.J., 1965.

1965 La Philosophie à l'heure du Concile (en collaboration avec S. Swiezawski, Préface d 'Olivier Lacombe), Paris : Société d'Editions Internationales, 1965.

Traduction polonaise : Filozofia w dobie soboru, Bibliothèque de Wiez, 86, Warszawa, 1995.

Traduction américaine : Philosophy during the second Vatican Council, Hardcover, 2005.

1960 Teoria poznania praktycznego (Théorie de la connaissance pratique), Lublin : TNKUL, 1960.

(un ouvrage de 139 pages, avec résumé en français, 133-138).

1949 Teoria reguly spolecznej Leona Duguit, Lublin : TNKUL, 1949.

(thèse de philosophie du droit sur Léon Duguit, avec un résumé en français).

\section{- Les articles de Jerzy Kalinowski}

1948a O tak zwanym neopozytywizmie prawnym, Przeglad Powszechny, 13, 1948.

1948b Problem stosowalnoci metody aksjomatycznej w nauce prawa, Sprawozdanie z dzialalnosci TFP $w$ Lublinie, Lublin, Towarzystwo filozofii praktycznej w Lublinie, 107-114, 1948.

(communication à la Société de Philosophie et de Psychologie de Lublin).

1949a Zagadnienie aksjomatyzacji nauki prawa, Roczniki Nauk Spolecznych, 1, 29-46, 1949. 
1949b Sp. Czeslaw Martyniak, Roczniki Nauk Spolecznych, 1, 19-21, 1949.

1953 Théorie des propositions normatives, Studia Logica, 1, 147-182, 1953.

(réédité dans le recueil Etudes de Logique déontique (1953-1969), 19-53, Paris : 1972).

1956 Polskie skrypty i podreczniki logiki dla prawnikow, Studia Logica, 4, 266-272, 1956.

1955-57 Interpretacja prawa a logika zdan normatywnych, Roczniki Filozoficzne, 5, 151-169, 1955-1957.

1958 O istocie i jednosci filozofii, Roczniki Filozoficzne, 6, 5-17, 1958.

1959a Y a-t-il une logique juridique?, Logique et Analyse, 2, 49-53, 1959.

1959b Interprétation juridique et logique des propositions normatives, Logique et Analyse, 2, 128-142, 1959.

(Version française de : [1955-57] Interpretacja prawa a logika zdan normatywnych, Roczniki Filozoficzne, 5, 151-169, 1955-1957.)

1959c La théorie aristotélicienne des habitus intellectuels, Revue des Sciences Philosophiques et Théologiques, 43, 248-260, 1959.

1960a Le droit naturel et la personne humaine, Chronique Sociale de France, 68, 195-199, 1960.

1960a Le fondement métaphysique de la morale, Actes du 4e Congrès des professeurs de philosophie, Facultés Catholiques de Lyon, 1-9, 1960.

1961 Métathéorie du système des règles de l'agir, Revue de l'Université d'Ottawa, 31, 183-212, 1961.

1962 Qu'est-ce que la philosophie du droit?, Archives de Philosophie du Droit, 7, 127-130, 1962.

1963a Lex et ius. A propos d'une définition du droit de Michel Villey, Archives de Philosophie du Droit, 8, 285-291, 1963.

1963b Esquisse de l'évolution d'une conception de la métaphysique, Recherches de philosophie, 6, 97-133, 1963.

1963c La aportación de la lógica contempoŕanea al estudio del carácter sistemático del derecho, Revista de Instituto de Ciencias Sociales, 35-55, 1963. 
1963d Au carrefour des métaphysiques : Pluralité des vues et indécisions dans la théorie de la philosophie d'Aristote, Revue des Sciences Philosophiques et Théologiques, 47, 545-570, 1963.

1963e La norme, l'action et la théorie des propositions normatives. Réponse à Ota Weinberger, Studia Logica, 14, 99-114, 1963.

(réédité dans le recueil Etudes de Logique déontique (1953-1969), Paris : $55-71,1972$ )

1963f Ontologia czy aitiologia ?, Znak, 15, 1069-1076, 1963.

1964a Logique et philosophie du droit subjectif, Archives de Philosophie du Droit, 9, 37-43, 1964.

1964b Contribution à la critique de la philosophie existentialiste du droit, Archives de Philosophie du Droit, 9, 257-272, 1964.

1964c Le syllogisme d'application du droit, Archives de Philosophie du Droit, 9, 273-285, 1964.

1964d Obligation dérivée et logique déontique relationnelle. Remarques sur le système de G. H. von Wright et sur le développement de la logique déontique, Notre-Dame Journal of Formal Logic, 5, 181-190, 1964.

(réédité dans le recueil Etudes de Logique déontique (1953-1969), Paris : 73-84, 1972.)

1964e Essai sur le caractère ontique du droit, Revue de l'Université d'Ottawa, 34, 81-99, 1964.

Metateoría del sistema normativo, Editorial Idearium, [s.d.], Mendoza.

(version espagnole de : 1961 Métathéorie du système des règles de l'agir, Revue de l'Université d'Ottawa, 31, 183-212, 1961.)

$1964 \mathrm{f}$ Sad o prawie i osobie, Znak, 16, 464-478, 1964.

1965a La logique des normes d'Edmund Husserl, Archives de Philosophie du Droit, 10, 107-116, 1965.

(réédité dans le recueil Etudes de Logique déontique (1953-1969), Paris, 1972, 111-122)

1965b Possibilité et structure de la logique déontique. A propos du livre de G. H. von Wright Norm and Action, Archives de Philosophie du Droit, 10, 313-333, 1965.

(réédité dans le recueil : Etudes de Logique déontique (1953-1969), Paris : 85-109, 1972.) 
1965c Les thèmes actuels de la logique déontique, Studia Logica, 17, 75-107, 1965.

(réédité dans le recueil Etudes de Logique déontique (1953-1969), Paris : 123-158, 1972. Version roumaine sous le titre de Temele actuele logicii deontice in Norme, Valori, Actiune, Bucuresti : 55$78,1979)$.

1965d Logique déontique et logique juridique, Les Etudes philosophiques, 20, 157-165, 1965.

1965e Jugement sur la personne et le droit. Méditation sur un thème de Simone Weil, Revue de l'Université d'Ottawa, 35, 265-275, 1965.

(version française de : 1964 Sad o prawie i osobie, Znak, 16, 464478, 1964.)

$1965 f$ Rozmowa o filozofii w okresie Soboru (avec Stefan Swiezawski), Znak, 17, (137-138), 939-955, 1965.

(Fragments de l'ouvrage : 1965 La Philosophie à l'heure du Concile (en collaboration avec S. Swiezawski, Préface d'Olivier Lacombe), Paris : Société d'Editions Internationales, 1965.)

1965g Do czytelnika "Znaku"(avec Stefan Swiezawski), Znak, 17 (137138), 1693-1696, 1965.

1966a De la spécificité de la logique juridique, Archives de Philosophie du Droit, 11, 7-23, 1966.

1966b Le bien, la morale et la justice. Sur The Varieties of Goodness de G.H. von Wright, Archives de Philosophie du Droit, 11, 311-328, 1966.

1966c La pluralité ontique en philosophie du droit, Revue philosophique de Louvain, 64, 263-280, 1966.

1966d Une nouvelle branche de la logique : la logique déontique, Sciences et techniques humaines, 6, 8-9, 1966.

$1966 \mathrm{e}$ W zwiazku z tzw. metafizyka egzystencjalna, Znak, 18 (142), 452-467, 1966.

1967a Logique formelle et droit, Annales de la Faculté de droit et des sciences économiques de Toulouse, 15, 197-211, 1967.

1967b Application du droit et prudence, Archiv für Rechts-und Sozialphilosophie, 53, 161-178, 1967.

1967c Le couple, notion de logique, Essais, 2, 27-29, 1967.

1967 L'axiomatisation et la formalisation de la théorie hexagonale de l'opposition de M. R. Blanché (système B), Les études philoso- 
phiques, 22, 201-209, 1967.

(réédité dans le recueil Etudes de Logique déontique (1953-1969), Paris : 159-167), 1972.

1967d La pluralité des philosophies et l'actualité de St. Thomas d'Aquin, Sapienza, 20, 155-163, 1967.

1967e Miedzynarodowe kolokwium filozofii prawa w Tuluzie, Ruch Filozo- ficzny, 26, 1-2, 1967.

1968a Kolokwium logiki prawa w Tuluzie, Studia Logica, 22, 165167, 1968.

1968b Derecho, lógica y lenguaje, Revista de la Universidad Externado de Colombia : (majo) 1968.

(réédité dans La Justicia, Mexico : noviembre 1969).

1968c La signification de la logique déontique pour la morale et le droit, Akten des XIV Internationalen Kongres für Philosophie, Wien (Herder) : 285-290, 1968.

(réédité dans le recueil Etudes de Logique déontique (1953-1969), Paris : 169-176, 1972.)

1968d Ontologie et esthétique chez Roman Ingarden, Archives de Philosophie, 31, 281-287, 1968.

1968e La logique des valeurs d'Edmund Husserl, Archives de Philosophie du Droit, 13, 267-282, 1968.

(réédité dans le recueil Etudes de Logique déontique (1953-1969), Paris : 237-256, 1972)

$1968 \mathrm{f}$ Saggi di logica giuridica (presentazione e traduzione a cura di P. de Lalla), II Foro Penale, 23, 305-341, 1968.

(traduction italienne des articles cités ci-haut : [Kalinowski 1959a] : Y a-t-il une logique juridique? , Logique et Analyse, 2, 49-53, 1959 et [Kalinowski 1959b] : Interprétation juridique et logique des propositions normatives, Logique et Analyse, 2, 128-142, 1959.)

$1968 \mathrm{~g}$ Un seminario sui rapporti tra sociologia del diritto e filosofia del diritto, Nuovi sviluppi della sociologia del diritto, 1 (19661967), a cura di R. Treves, Milano : Ed. Comunità, 125-138, 1968.

$1968 \mathrm{~h}$ Sur la vérité des normes, Rivista internazionale di filosofia del diritto, 45, 592-602, 1968.

1968i Le chrétien et la philosophie, Roczniki Filozoficzne, 16, 17-35, 1968. 
1969a Intervention de Georges Kalinowski, La logique judiciaire, 5e Colloque des Instituts d'études judiciaires, Paris, du 18 au 20 mai 1967, Paris : PUF, 127-128, 1969.

1969b Note critique sur la logique déontique d'Alf Ross, Archiv für Rechts-und Sozialphilosophie, 55, 41-72, 1969.

(réédité dans le recueil Etudes de Logique déontique (1953-1969), Paris : 187-211, 1972.)

1969c La logique des lacunes en droit, Archives de Philosophie du Droit, 14, 353-362, 1969.

1969d De la signification de la philosophie du temps pour la philosophie du droit, Ius, 20, 191-294, 1969.

1969e Uczen i mistrz (Le disciple et le maître). A propos des lettres d'Edmund Husserl à R. Ingarden, Kultura, 9 (264), 140-145, 1969.

$1969 \mathrm{f}$ Note sur le rapport entre le fait et le droit, Rivista internazionale di filosofia del diritto, 46, 411-422, 1969.

$1969 \mathrm{~g}$ Sur les syllogismes méréologiques. Une contribution à la logique déontique, Rozprawy Filozoficzne, 119-125, 1969.

(réédité dans le recueil Etudes de Logique déontique (1953-1969), Paris : $177-186,1972$.)

1970a Chrzescijanin i filozofia, W nurcie zagadnien posoborowych, IV, Warszawa (Wydawnictwo SS. Loretanek-Benedyktynek), 2341, 1970.

(version polonaise du : 1968 Le chrétien et la philosophie, Roczniki Filozoficzne, 16, 17-35, 1968.)

1970b Les thèses principales de l'esthétique ingardénienne, Archives de Philosophie, 33, 945-950, 1970.

1970c Théorie, métathéorie ou philosophie du droit. Réflexions sur The Concept of Law de H. L. A. Hart et On Law and Justice d'Alf Ross, Archives de Philosophie du Droit, 15, 179-195, 1970.

1970d O nauczaniu logiki na wydzialach prawa, Studia Logica, 26, 115-12, 19702.

1970e Sur l'enseignement de la logique dans les facultés du droit, Archives de Philosophie du Droit, 15, 310-329, 1970.

(version française modifiée du : 1970 O nauczaniu logiki na wydzialach prawa, Studia Logica, 26, 115-12, 1970.)

1970 Une théorie de la dogmatique juridique. Note sur J. Kmita et L. Nowak, Archives de Philosophie du Droit, 15, 405-413, 1970. 
1970 Le raisonnement juridique et la logique juridique, Logique et Analyse, 13, 1-16, 1970.

$1970 \mathrm{~g}$ Pour une conception adéquate de la logique déontique, $P o-$ lish Congress of Contemporary Science and Culture in Exil, vol. I, London : 67-73, 1970.

1970h Philosophie, théologie et métathéorie, Recherches de philosophie, 8, 157-205, 1970.

1970i L'intuitionisme en logique déontique, Rechtstheorie, 1, 157182, 1970.

1971a Une nouvelle branche de la logique : la logique déontique, Archives de Philosophie, 34, 3-36, 1971.

1971b La logique juridique à la veille du congrès bruxellois, Archives de Philosophie du Droit, 16, 365-381, 1971.

1971c O prawdzie (Sur la vérité). A l'occasion de la traduction française de l'œuvre d'A. Tarski, Kultura, 1(280)-2 (281), 191-198, 1971.

1971d Sur la syllogistique déontique de Z. Ziemba, Studia Logica, 29, 125-142, 1971.

(réédité dans le recueil Etudes de Logique déontique (1953-1969), Paris : 213-235, 1972.)

1972a Le raisonnement juridique : état actuel de la question, Archiv für Rechts-und Sozialphilosophie, Beiheft 7 : Die juristische Argumentation, 17-42, 1972.

1972b Le problème des rapports entre la sémantique et la pragmatique, in G. Kalinowski, L. Koj, La sémantique et la pragmatique, Archives de Philosophie, 35, 177-182, 1972.

1972c Philosophie et logique de l'interprétation en droit, Archives de Philosophie du Droit, 17, 39-49, 1972.

1972d Théorie des normes, logique déontique, ontologie juridique, in G. Kalinowski, M. Villey et J.-L. Gardies, « Autour de l'a priori de la rationalité déontique. Dialogue à trois voix à propos de l'ouvrage de J.-L. Gardies Essai sur les fondements a priori de la rationalité morale et juridique », Archives de Philosophie du Droit, 17, 397406, 1972.

1972e Sur l'homme et l'aristotélisme chrétien, La pensée catholique, 140, 20-25, 1972.

1972 f Remarques préliminaires, in G. Ka1inowski, L. Koj, « Le statut sémiotique des guillemets et leur antinomie. A propos de Le concept 
de vérité dans les langages formalisés d'Alfred Tarski », Les études philosophiques, 27, 239-250, 1972.

1972g Le rationnel et l'argumentation, Revue philosophique de Louvain, 70, 404-418, 1972.

1972h Préface in J.-L. Gardies, Essai sur les fondements a priori de la rationalité morale et juridique, Paris : L.G.D.J., 1972.

1972i L'expérience de la foi et l'existence de Dieu, Revue philosophique de Louvain, 70, 620-634, 1972.

1973a Intervention à la XXIXe Semaine de Synthèse, Actes de la XXIX ${ }^{e}$ Semaine de Synthèse : Le droit, les sciences humaines et la philosophie, Paris : Vrin, 257-258, 1973.

1973b Norm and Logic, The American Journal of Jurisprudence, 18, 165-197, 1973.

1973c La logique et son histoire, Archives de Philosophie, 36, 121126, 1973.

1973d Le fondement objectif du droit d'après la Somme de Théologie de saint Thomas d'Aquin, Archives de Philosophie du Droit, 18, 59-75, 1973.

1973e Sur l'emploi métonymique du terme ius par Thomas d'Aquin et sur la muabilité du droit naturel chez Aristote, Archives de Philosophie du Droit, 18, 331-339, 1973.

$1973 \mathrm{f}$ A propos de Vingt années de recherches déontiques de J.-L. Gardies, Archives de Philosophie du Droit, 18, 425-432, 1973.

1973g O logice norm, Kultura, 304-305, 202-208, 1973.

1973h Traktat o sprawiedliwosci i innych cnotach spolecznych, $\mathrm{Pr}$ zeglad Powszechny, 5 (309), 10-12, 1973.

1973i La logique de Lesniewski et la théologie de St. Anselme, Archives de Philosophie, 36, 407-416, 1973.

1973j A propos de la méta-éthique. Discussion avec Tadeusz Styczen, Rivista di filosofia neoscolastica, 65, 794-806, 1973.

1973k Convicciones y fonctores de aceptación. Al margen de una reciente contribución a los fundamentos lógicos de las convicciones, Ethos, 1, Buenos Aires : 227-234, 1973.

1973m Rozum, rozsadek i filozofia, Studia Philosophiae Christianae, 9 (1), 29-54, 1973.

1973n Doswiadczenie wiary a istnienie Boga, Studia z filozofii Boga, II, Warszawa : ATK, 323-338, 1973. 
1973p Filozofia, wiedza czy przekonanie?, Z zagadnien kultury chrzescijanskiej, Lublin : TNKUL, 219-230, 1973.

1974a 0 czlowieku i arystotelizmie chrzescijanskim, O cz1owieku dzis, Warszawa: Wydawnictwo SS. Loretanek-Benedyktynek, 119-125, 1974.

(version polonaise du : 1972 Sur l'homme et l'aristotélisme chrétien, La pensée catholique, 140, 20-25, 1972.)

1974b L'homme : âme et matière. A propos du Problème de l'âme de Cl. Tresmontant, Archives de Philosophie, 37, 411-433, 1974.

1974c Raison, entendement et philosophie, Dialogue, 13, Montréal : 123-138, 1974.

(version française du : 1973 Rozum, rozsadek i filozofia, Studia Philosophiae Christianae, 9 (1), 29-54, 1973.)

1974d Czlowiek: materia i dusza, O czlowieku dzis, Warszawa: Wydawnictwo SS. Loretanek-Benedyktynek, 95-118, 1974.

(version polonaise du : 1974 L'homme : âme et matière. A propos du Problème de l'âme de Cl. Tresmontant, Archives de Philosophie, 37, 411-433, 1974.)

1974e La logique des prédicats déontiques de Leibniz, in G. Kalinowski, J.-L. Gardies, « Un logicien déontique avant la lettre. Godtfried Wilhelm Leibniz », Archiv für Rechts-und Sozialphilosophie, 60, 81-98, 1974.

1974f La philosophie : savoir ou croyance?, Revue de l'Université d'Ottawa, 44, 396-405, 1974.

(version française du : 1973 Filozofia, wiedza czy przekonanie?, Z zagadnien kultury chrzescijanskiej, Lublin : TNKUL, 219-230, 1973.)

1974g Sur les langages respectifs du législateur, du juge et de la loi, in M. Villey, G. Kalinowski, J.-L. Gardies, «Indicatif et impératif juridiques. Dialogue à trois voix », Archives de Philosophie du Droit, 19, 63-74, 1974.

1974h La logique juridique, la sémiotique et la rhétorique. A propos de Law and Logic de J. Horovitz, Archives de Philosophie du Droit, 19, 455-469, 1974.

1974i Über die deontischen Funktoren, Normenlogik, H. Lenk ed., Pullach bei Munchen : Verlag Dokumentation, 39-63, 1974.

$1974 \mathrm{j}$ Sur quelques suggestions en logique modale et en logique trivalente, Logique et Analyse, 17, 111-125, 1974. 
1974k La philosophie de Saint Thomas d'Aquin face à la critique de la métaphysique par Kant, Nietzsche et Heidegger, Studi Tomistici, III, San Tommaso e il pensiero moderno, Roma (Città Nuova Editrice) - Accademia Romana di S. Tommaso d'Aquino e di religione cattolica, 257-283, 1974.

$1974 \mathrm{~m}$ Sur un nouveau système de logique déontique, Logique et Analyse, 17, 343-387, 1974.

1974n Mit mitu?... Uwagi o ksiazce L. Kolakowskiego Obecnosc mitu, Paris : 1972, Zeszyty Naukowe KUL, 18 (3), 107-112, 1974.

(version polonaise de la seconde partie de l'article : 1975 Métaphysique : science ou mythe?, Archives de Philosophie, 38, 239-252, 1975, répertorié plus bas, rééditée dans Przeglad Powszechny, 2, 8-13, 1974.)

1975a Metafizyka i fenomenologia osoby ludzkiej, Analecta Cracoviensia, 5-6 (1973-1974), 63-71, 1975. (réédité en français dans le recueil Autour de Personne et Acte de Karol Wojtyla, Aix-enProvence : Presses Universitaires d'Aix-Marseille, 35-45, 1987)

1975b Métaphysique : science ou mythe?, Archives de Philosophie, 38, 239-252, 1975.

1975c Sur la présentation de la logique mathématique dans une récente publication. Quelques remarques à propos de Mathematische Logik de W. Strombach, H. Emde et W. Reyersbach, Archiv für Rechts-und Sozialphilosophie, 61, 117-124, 1975.

1975d Théorie des directives, méthodologie des sciences juridiques, théorie du droit. Sur quelques publications polonaises récentes, Archives de Philosophie du Droit, 20, 367-384, 1975.

1975e De la philosophie hégélienne du droit et de l'Etat. Note sur la thèse de J. Ph. Guinle, Archives de Philosophie du Droit, 20, 351-365, 1975.

$1975 \mathrm{f}$ Du métalangage en logique. Réflexions sur la logique déontique et son rapport avec la logique des normes, Documents de travail et prépublications, Urbino (Université d'Urbino), Centre International de Sémiotique et de Linguistique, A (48), 1-28, 1975.

$1975 \mathrm{~g}$ Logica del diritto. Lineamenti generali, Enciclopedia del diritto, vol. 25, Milano : Giuffré Editore, 7-13, 1975.

1976a L'Université catholique de Lublin et la philosophie en Pologne, Rivista di filosofia neoscolastica, 68, 645-657, 1976. 
1976b De la philosophie pratique à la logique déontique, Saint Thomas d'Aquin pour le septième centenaire de sa mort. Essais d'actualisation de sa philosophie, sous la rédaction de S. Kaminski, M. Kurdzialek, Z. J. Zdybicka, Lublin : Towarzystwo Naukowe Katolickiego Uniwersytetu Lubelskiego (TNKUL), 223-239, 1976.

1976c Le sens du discours métaphysique et les premiers principes, Rivista di filosofia neoscolastica, 68, 3-19, 1976.

1976d Un aperçu élémentaire des modalités déontiques, Langages, 10, 10-18, 1976.

1976e La personne humaine et le droit naturel. Un métaphysicien parle de l'homme et du droit, Archives de Philosophie du Droit, 21, 239-248, 1976.

1976f O poslugiwaniu sie w logice deontycznej jezykami preskryptywnym i deskryptywnym. Dwa pytania na temat "derived obligation", Studia Filozoficzne, 3, nr. specjalny w 90 rocznice urodzin Profesora Tadeusza Kotarbinskiego, 121-128, 1976.

1977a Über die Bedeutung der Deontik für Ethik und Rechtsphilosophie, Deontische Logik und Semantik, (éd.). A. C. Conte, R. Hilpinen, G. H. von Wright, Wiesbaden (Athenaion), 101-130, 1977.

(traduction italienne : "Il significato della deontica per la filosofia morale e guiridica ", Logica deontica e semantica, a cura di G. di Bernardo, Bologna (Il Mulino), 251-289, 1977; version castillane, voir le livre : 1978 Logica de las normas y 1ogica deontica. Posibilidad y relaciones, Numéro spécial des Cuadernos de Metodologia y filosofia del derecho, 1, Valéncia (Venezuela) : Universidad de Carabobo, 1978.)

1977b La grammaire pure et les catégories sémantiques. A propos d'Esquisse d'une grammaire pure de J.-L. Gardies, Archives de Philosophie, 40, 467-475, 1977.

1977c La razón práctica : sus conceptos, judicios y razonamientos, Anales de la cátedra Francisco Suares (Granada), 17, 201-216, 1977.

1977d La logique juridique de Leibniz. Conception et contenu, Studia Leibniziana, 9, 168-189, 1977.

1977e Autorytet w filozofii, Zeszyty Naukowe, KUL, 20, (3-4), 53-58, 1977.

$1977 f$ Sur le langage de la confession chrétienne de la foi, AA.VV. La confession de la foi, textes réunis par Cl. Bruaire, Paris : Fayard, 231-246, 1977. 
$1977 \mathrm{~g}$ Du prescriptif et du descriptif en logique déontique. Deux questions à propos de l'obligation dérivée, Logique et Analyse, 20, 317-328, 1977.

(version française du : [1976] «O poslugiwaniu sie w logice deontycznej jezykami preskryptywnym i deskryptywnym. Dwa pytania na temat "derived obligation", Studia Filozoficzne, 3, nr. specjalny w 90 rocznice urodzin Profesora Tadeusza Kotarbinskiego, 121-128, 1976. »)

1977h W zwiazku z podzialem metafizyki na ogolna i szczegolowa, Studia z filozofii Boga, III, Warszawa : ATK, 70-78, 1977.

1978a Logique et méthodologie juridique. Réflexions sur la rationalité formelle et non formelle en droit, Archives de Philosophie du Droit, 23, 59-68, 1978.

1978b Sur l'antinomie de Cervantes. Etude de sémiotique juridique, Archives de Philosophie du Droit, 23, 325-332, 1978.

$1978 \mathrm{c}$ Note sur l'informatique juridique. A propos des récentes publications de l'I.R.T.I.J., Archives de Philosophie du Droit, 23, 381383, 1978.

1978d Przekonania i funktory przekonaniowe. Uwagi na marginesie Podstaw logicznej teorii przekonan W. Marciszewskiego, Studia z teorii poznania $i$ filozofii wartosci, Wroclaw-Warszawa-Krakow (Ossolineum), 59-70, 1978.

(version polonaise du : 1973 Convicciones y fonctores de aceptación. Al margen de una reciente contribución a los fundamentos lógicos de las convicciones, Ethos, 1, Buenos Aires : 227-234, 1973.)

1978e Rapport sur la recherche en philosophie du droit : 1973-1976. La recherche en sciences humaines 1976-197\%. Bilan et perspectives, Paris : (CNRS), 97-99, 1978.

$1978 \mathrm{f}$ Rechtslogik und die Logik der Präferenz, Strukturierung und Entscheidungen in Rechtsdenken (Forschungen aus Staat und Recht, 43), I. Tammelo und H. Schreiner ed., Wien, New York : Springer, 31-40, 1978.

1978g Zu den Rechtslogischen Stellungsnahmen von Thomas Cornides, Strukturierung und Entscheidungen in Rechtsdenken (Forschungen aus Staat und Recht, 43), I. Tammelo und H. Schreiner ed., Wien, New York : Springer, 265-269, 1978.

(Ce volume contient aussi, aux pages 271-275, une réponse de Cornides à Kalinowski.) 
1978h O filozofii i filozofowaniu. W zwiazku z nauczaniem filozofii w uczelniach katolickich. Wspomnienia i uwagi, Zeszyty Naukowe KUL, 21 (2), 41-48, 1978.

1978i Les causes de certaines antinomies juridiques. Réflexions inspirées par la lecture de Leibniz, Logique et Analyse, 21, 89-110, 1978.

1978j Réponse à A. G. Conte, Logique et Analyse, 21, 121-122, 1978.

1979a O antynomii Cervantesa, Studia semiotyczne, IX (Ossolineum), 197-204, 1979.

(version polonaise du : 1978 Sur l'antinomie de Cervantes. Etude de sémiotique juridique, Archives de Philosophie du Droit, 23, 325$332,1978$.

1979b L'interprétation du droit et la sémantique. A propos des travaux de J. Wolenski et T. Gizbert-Studnicki, Archives de Philosophie du Droit, 24, 363-370, 1979.

1979c Autour de l'organisation contemporaine du pensable. A propos de Foi et raison logique et Du principe de Breton, Les études philosophiques (article rédigé et rendu à la Revue en 1972), 1, 5578, 1979.

1979d Za przykladem Etyki Nikomachejskiej, Roczniki Filozoficzne, 27, 155-158, 1979.

1980a Zur Semantik der Rechtssprache, Rechtstheorie, Beiheft 1 : Argumentation und Hermeneutik in der Jurisprudenz, 239-252, 1980.

1980b La praxéologie de T. Kotarbinski, Archives de Philosophie, 43, 453-463, 1980.

1980c Loi juridique et loi logique. Attribution à la sémantique de la loi juridique, Archives de Philosophie du Droit, 25, 123-136, 1980.

1980d Une mise en question de la logique des normes. En réponse aux objections de H. Kelsen, Archives de Philosophie du Droit, 25, 345-365, 1980.

1980e Humanisme théocentrique. L'enseignement de Jean-Paul II sur l'homme, Seminarium, 20, 130-144, 1980.

(réédité en français dans le recueil Autour de Personne et Acte de Karol Wojtyla, Aix-en-Provence : Presses Universitaires d'AixMarseille, 1987)

1980 f Karol Wojtyla face à Max Scheler ou l'origine de Osoba i czyn, Revue thomiste, 80, 456-465, 1980. 
(réédité en français dans le recueil Autour de Personne et Acte de Karol Wojtyla, Aix-en-Provence : Presses Universitaires d'AixMarseille, 19-31, 1987.)

$1980 \mathrm{~g}$ Sur la réduction de la logique déontique à la logique modale aléthique, Mélanges Tsatsos, Athens (A. N. Sakkoulas Juristischer Verlag), 653-666, 1980.

1980h Sur l'inférence par analogie (texte reprographié), Equipe de recherche CNRS. Groupe de travail sur l'analogie. Recueil de Textes, $2,1-8,1980$.

1980i Le raisonnement en sciences juridiques, Représentation des connaissances et raisonnement dans les sciences de l'homme, M. Borillo éd., Paris : Institut National de Recherche en Informatique et en Automatique, 476-487, 1980.

1980j Sur les universaux, Le bulletin du groupe de recherches sémiolinguistiques de l'Ecole des Hautes Etudes en Sciences Sociales (EHESS), CNRS, 14, 20-28, 1980.

1980k Logique juridique de Leibniz. Conception et contenu. Résumé, Akten des III Internationalen Leibniz Kongresses, 7-17 Nov. 1977 Hannover, Bd. III : Logik, Erkenntnisstheorie, Wissenschaftstheorie, Metaphysik, Theologie, Wiesbaden (Franz Steiner Verlag), 1213, 1980 .

1980m Carré sémiotique et carré logique, Le bulletin du groupe de recherches sémiolinguistiques de l'Ecole des Hautes Etudes en Sciences Sociales, (EHESS) CNRS, 17, 5-9, 1980.

1980n Prefacio (trad. Roque Carrion Wam) à E. Oppenheim, Lineamientos de un análisis lógico del derecho, (Cuadernos de metodología y filosofía del derecho, 4), Valencia (Venezuela) : 11-12, 1980.

$1980 \mathrm{~m}$ Sobre las semánticas de los mundos possibles para los sistemas de lógica deóntica, Teorema, 10, 307-325, 1980.

1981a Teorema entrevista a Georges Kalinowski, Teorema, 11 (4), 315-333, 1981.

(interview sur la situation de la logique en Pologne, traduite en castillan par Adoración Sanchéz Garcia).

1981b La justification de la morale naturelle, AA.VV., La morale, Sagesse et salut, Cl. Bruaire (éd.), Paris : Fayard, 209-220, 1981.

1981c Obligations, permissions et normes. Réflexions sur le fondement métaphysique du droit, Archives de Philosophie du Droit, 26, 331-343, 1981. 
1981d Sur les sémantiques des mondes possibles pour les systèmes de logique déontique, Logique et Analyse, 24, 81-98, 1981.

(version française du : 1980 Sobre las semánticas de los mundos possibles para los sistemas de lógica deóntica, Teorema, 10, 307$325,1980$.

1981e Discours de louange et discours métaphysique. Denys l'Aréopagite et Thomas d'Aquin, Rivista di filosofia neoscolastica, 73, 399-404, 1981.

1982a Les sciences sociales et la philosophie, Epistemologia $5(\mathrm{Nu}-$ méro spécial : Atti del Convegno Fundations of social sciences, Trento : 1980), 117-136, 1982.

1982b Vérité analytique et vérité logique, Actes sémiotiques - Documents du groupe de recherches sémio-linguistiques (EHESS) CNRS IV, 40, 5-27, 1982.

1982c Die Rhetorik des Aristoteles und die juristische Logik : zum formalen Charakter der rhetorischen Beweisführung. Rhetorische Rechtstheorie (Theodor Viehweg zum 75. Geburstag), O. Ballweg und Th.-M. Seibert (ed.), Freiburg-München : Verlag Karl Alber, 99-110, 1982.

1982d La logique juridique et son histoire, Archives de Philosophie du Droit, 27, 275-289, 1982.

(réédité en Anuario Filosófico, 16, 331-350, 1983)

1982e En réponse à Jean-Yves Lacoste autour de The Act and Person, Revue Thomiste, 82, 626-633, 1982.

(réédité en français dans le recueil Autour de Personne et Acte de Karol Wojtyla, Aix-en-Provence : Presses Universitaires d'AixMarseille, 71-82, 1987.)

1983a Deux espèces de sémantique pour la logique modale, $\mathrm{La} \mathrm{No-}$ tion sémantico-logique de modalité, (Actes du Colloque de Metz du 5 au 7 novembre 1981), J. David et G. Kleber éds., Paris (Klincksieck), 25-41, 1983.

1983b Logique juridique : Conceptions et recherches, Rechstheorie, 14, 1-17, 1983.

(édition d'une conférence de 1978 à l'ENS de Paris)

1983c Notions de Nature. Sur la muabilité du concept de nature et l'immuabilité de la loi naturelle, Das Naturrechtsdenken heute und morgen. Gedächtnisschrift für René Marcic, D. Mayer-Maly und P.M. Simons (eds.), Berlin : Duncker und Humblot, 45-55, 1983. 
1983d Il diritto alla vita in Tommaso d'Aquino, Rivista di filosofia, 25-27, 43-61, 1983.

1983e Des divers sens du terme « jugement», (Actes du Colloque de philosophie du droit, Paris juin 1982), Archives de Philosophie du Droit, 28, 295-302, 1983.

$1983 \mathrm{f}$ La genèse d'un système de logique des normes, Informatica $e$ diritto, 9, 251-267, 1983.

1983g Leibniz et les sémantiques des mondes possibles, Leibniz Werk und Wirkung, IV Internationaler Leibniz Kongress - Hannover : 336-349, 1983.

1983h Préface à C.I. Massini, La prudencia juridica, Buenos Aires : Abeledo-Perrot, 13-17, 1983.

1984a Edith Stein et Karol Wojtyla sur la personne, Revue philosophique de Louvain, 82, 545-561, 1984.

(réédité en français dans le recueil Autour de Personne et Acte de Karol Wojtyla, Aix-en-Provence : Presses Universitaires d'AixMarseille, 85-100, 1987)

1984b Le fondement de la vie sociale de l'homme. Individu, personne, société, Doctor Communis, 123-134, 1984.

1984c Logique et ontologie, Archives de Philosophie, 47, 625-649, 1984.

1984d Sur l'objectivité de la pensée juridique, Objektivierung des Rechtsdenkens. Gedächtnisschrift für Ilmar Tammelo, Berlin, 267272, 1984.

1984e La mobilité du droit naturel chez Aristote et Thomas d'Aquin, Archives de Philosophie du Droit, 29, 187-190, 1984.

(traduction castillane : «De lo mutable y lo inmutable en el Derecho Natural $»$, Des ponennas sobre la mutabilidad del derecho natural on Aristotels y Thomas de Aquino, Prudentia iuris, 15, 92-94, 1985).

$1984 \mathrm{f}$ Logique déontique, Encyclopédie philosophique, Paris : PUF, 1984.

1984g Logique juridique, Encyclopédie philosophique, Paris : PUF, 1984.

1984h Réponse à l'enquête des Cuadernos de filosofía del derecho, Doxa, Problemas abiertos en filosofía de derecho, I, Alicante, 113128, 1984. 
1984i Présupposition, vérité et normes, Theorie der Normem. Festgabe fur Ota Weinberger zum 65. Geburtstag, Berlin (Duncher und Humbolt), 393-406, 1984.

1984j Izydora Dambska (1904-1983), Les études philosophiques, 3, 423-425, 1984.

1985a Le droit à la vie chez Thomas d'Aquin, Archives de Philosophie du Droit, 30, 315-330, 1985.

(version française du : [Kalinowski 1983d].)

1985b Avant-Propos à Les fondements logiques de la pensée normative. Actes du colloque de logique déontique de Rome (les 29 et 30 avril 1983), Analecta Gregoriana, VII-IX, 1985.

1985c Logique des normes, logique déontique et fondements de la pensée normative, Les fondements logiques de la pensée normative, Actes du colloque de logique déontique de Rome (les 29 et 30 avril 1983), Analecta Gregoriana, 147-174, 1985.

1985d Sobre la mutabilidad del concepto de naturaleza y la inmutabilidad de la ley naturel, Los derechos humanos, C.I. Massini (ed.), Mendoza : Editiorial Idearium, 97-110, 1985.

1985e Logique juridique et logique déontique, Revue de synthèse, 118-119, 229-244, 1985.

1985 f Filozofia prawa a antropologia filozoficzna, Problemy teorii $i$ filozofii prawa, Lublin : UMCS, 109-115, 1985.

$1985 \mathrm{~g}$ Sur le fondement des normes et des énoncés normatifs. A propos des idées de Von Wright et de Castaneda, Theoria, 1, 59-85, 1985 .

1985h Sur l'importance de la logique déontique pour la philosophie du droit, Rivista internazionale di filosofia del diritto, 62, 212-226, 1985.

1985 i Aristote et Thomas d'Aquin vus par M. Villey, Droit, Nature, Histoire - Michel Villey, Philosophe du droit. Actes du IVe Colloque de l'Association Française de Philosophie du Droit (Université de Paris II, du 23 au 24 novembre 1984), Presses Universitaires d'Aix-Marseille, 57-64, 1985.

1985j Sur l'argument ontologique de Leibniz, Studia Leibniziana, 17 (1), 94-97, 1985.

$1985 \mathrm{k}$ L'interprétation du droit : ses règles juridiques et logiques, Archives de Philosophie du Droit, 30, 171-180, 1985.

$1985 \mathrm{~m}$ Concepts et distinctions pour une sémiotique réaliste, Exi- 
gences et perspectives de la sémiotique. Recueil d'hommages pour Algirdas-J. Greimas, H. Parret et H. G. Ruprecht éds., AmsterdamPhiladelphia (John Benjamins Publishing Company), 25-39, 1985.

1985n La semiótica jurídica. Para una teoría de las funciones de las expresiones linguísticas , Cuadernos de semiótica juridíca, Universidad de Carabobo, Valencia (Venezuela) : CELIJS, 1985.

(traduction en castillan, par R. Carrion de : 1986 La logique des normes, Cahiers S.T.S. (Science - Technologie - Société), 12, Ordre juridique et ordre technologique, Paris : Editions du C.N.R.S., 27$38,1986$.

1986a La sémiotique juridique, Revue de la recherche juridique. Droit prospectif 2, 111-168, 1986.

1986b La logique des normes, Cahiers S.T.S. (Science - Technologie - Société), 12 : Ordre juridique et ordre technologique, Paris : Editions du C.N.R.S., 27-38, 1986.

1986c La réforme du thomisme et de la phénoménologie chez Karol Wojtyla selon Rocco Buttiglione, Archives de philosophie 39, 127146, 1986.

(réédité en français dans le recueil Autour de Personne et Acte de Karol Wojtyla, Aix-en-Provence : Presses Universitaires d'AixMarseille, 101-123, 1987.)

$1986 \mathrm{~d}$ Avant-propos à Pour une approche sémiotique et normative du droit, Actes sémiotiques. Documents 8 (71), 3-5, 1986.

1986e Introduction à Cypriani Regneri, Demonstratio logicae verae iuridica, Bologna : C.L.U.E.B., XI-XXVIII, 1986.

1987a La pensée philosophique de Jean-Paul II et la faculté de philosophie de l'Université Catholique de Lublin, Autour de Personne et acte de Karol Cardinal Wojtyla. Une rencontre de la phénoménologie et du thomisme, Aix : Presses Universitaires d'Aix-Marseille, 47-68, 1987.

(Il s'agit d'une réponse à l'article de J. Seifert, « Karol Cardinal Wojtyla (Pope John Paul II) as Philosopher and the Cracow/Lublin School of Philosophy », Aletheia. An International Journal of Philosophy, 11, Theme : Epistemology, 130-199, 1981.)

1987b Edmund Husserl sur les normes, Formalismus und Phänomenologie in Rechtsdenken der Gegenwart, Festgabe für Alois Troller zum 80. Geburtstag, Berlin : Duncker und Humblot, 185-200, 1987. 
1987c Sur les normes et leur logique. Remarques en marge de "Is and Ought"de Georg Henrik von Wright, Archives de Philosophie du Droit, 32, 277-289, 1987.

1987d O normach i ich logice. Uwagi na marginesie artykulu von Wright' Is and Ought, Roczniki Filozoficzne, 35, fasc. 1, 71-86, 1987.

(version polonaise du : [1987] « Sur les normes et leur logique. Remarques en marge de "Is and Ought"de Georg Henrik von Wright, Archives de Philosophie du Droit, 32, 277-289, 1987.»)

1988a Impératif, Dictionnaire encyclopédique de théorie et de sociologie du droit, sous la direction de A.-J. Arnaud et al., (Publié avec le concours du C.N.R.S.), Paris : 182-183, 1988.

1988b La logique aléthico-déontique et la présupposition, Studia Leibniziana, Sonderheft 15 : Leibniz : Questions de logique, Stuttgart (Franz Steiner Verlag)/ Wiesbaden : 72-81, 1988.

1988c La logique floue ou la logique formelle? ou Plaidoyer en faveur d'un enseignement de logique aux étudiants de droit, Recueil Dalloz Sirey, Hebdomadaire numéro 40, du ${ }^{\text {er }}$ décembre 1988, 297-298.

1988d Sur la distinction entre le descriptif et le normatif. Remarques à propos de l'Erreur de Hume de Jean-Louis Gardies, La philosophie du droit aujourd'hui, Archives de Philosophie du Droit, 33, 387-397, 1988.

$1988 \mathrm{e}$ Le droit et les actes de langage, The Review of Comparative Law, 2, 9-26, 1988.

$1988 \mathrm{f}$ Les performatifs en droit. Sur la distinction entre le langage prescriptif et le langage performatif, Normative Structures of the Social World, G. di Bernardo (ed.), Amsterdam : Rodopi, 205-221, 1988.

(Communication au séminaire organisé en 1983 à l'Università degli Studi di Trento)

1989a Aristote et la notion de présupposition. En complément à Présupposition, vérité et normes, Rechtstheorie - Zeitschrift fur Logik, Methodenlehre, Kybernetik und Soziologie des Rechts, 20 (4), 449450, 1989.

1989b Ontique et déontique, Rivista internazionale di filosofia del diritto, 56 (IV), 438-446, 1989.

1990a Trois notions de droit, Droits - Revue française de théorie juridique, 10, 43-46, 1990. 
1990b Ontic and Deontic, Revue internationale de sémiotique juridique (International Journal for the Semiotics of Law) 3 (8), 133146, 1990.

1991 Octavian Jonescu (1910-1990), Arch. de Philosophie du Droit, 36, 333-334, 1991.

1991-92 Dowody na nieistnienie przedmiotow ogolnych, Roczniki Filozoficzne, 39-40, fasc. 1, 65-78, 1991-1992.

1992a De la signification des normes juridiques, Revue internationale de sémiotique juridique, 5 (1), 131-136, 1992.

$1992 \mathrm{~b}$ Sur les normes et la relation normative. Contribution à la sémiotique du langage normatif et à la logique des normes, Zeszyty Naukowe Wyzszej Szkoly Pedagogicznej im. Powstancow Slaskich w Opolu, Matematyka, XXVIII, 57-70, 1992.

1992c De la signification des normes juridiques. A propos de l'article de Jerzy Wroblewski The Problem of the Meaning of the Legal Norm, Revue internationale de sémiotique juridique, 5 (14), 131136, 1992.

1993 Ku probie konstrukcji metafilozofii, Studia metafilozoficzne, I : Dyscypliny i metody filozofii, A. B. Stepien i T. Szubka ed., Lublin : TNKUL, 327-333, 1993.

(réimpression de l'exposé tenu à KUL le 13 juin 1953 r., et publié dans : "Sprawozdania z Czynnosci Wydawniczej i Posiedzen Naukowych oraz Kronika Towarzystwa Naukowego KUL", 6, 76-80, 1952-1953).

1995 Zdania modalne "de re"i "de dicto". Przyczynek do porownania ujecia sredniowiecznego i wspolczesnego, in Miedzy logika a etyka. Prace ofiarowane Profesorowi Leonowi Kojowi, Lublin : Wydawnictwo UMCS, 19-27, 1995.

1995-96a Les démonstrations de la non-existence des objets généraux chez Lesniewski, Stanislaw Lesniewski aujourd'hui, Recherches sur la philosophie et le langage, 16, D. Miéville et D. Vernant éds., Neuchâtel : 121-145, 1995-1996.

1995-96b O istotnej uzytecznosci ontologii Stanislawa Lesniewskiego, Summarium, 24-25, 44-45, 1995-1996.

2001 La logique des normes et Aristote, Logique et Métaphysique dans l'Organon d'Aristote, J. Follon et M. Bastit éds., Louvain (Peeters), 93-104, 2001. 
2002 Metametafizyka. Przyczynek do rozwazan nad koncepjami metafizyki, Studia metafilozoficzne, II, Lublin : TNKUL, 7-18, 2002.

\section{- Choix de recensions ${ }^{1}$}

1973 Archives de Philosophie, 36, 660-664, 1973 :

Alchourron, C. \& Bulygin, E, Normatives Systems.

1972 Archives de Philosophie du Droit, 17, 524-525, 1972 :

Bernardo, G. di, Introduzione alla logica dei sistemi normativi.

1971 Archives de Philosophie, 34, 157-160, 1971 :

Blanché, R., Raison et discours.

1967 Les études philosophiques, 22, 222, 1967 :

Blanché, R., La science actuelle et le rationalisme.

1966 Les études philosophiques, 21, 541-542, 1966 :

Blanché, R., Structures intellectuelles.

1963 Archives de Philosophie du Droit, 8, 298-317, 1963 :

Bobbio, N. Trends in Italian Legal Theory.

1966 Les études philosophiques, 21, 542-543, 1966 :

Bobbio, N., Giusnaturalismo e positivismo giuridico.

1970 Archives de Philosophie, 33, 135-137, 1970 :

Bochenski, J. M., The Logic of Religion.

1971 Archives de Philosophie du Droit, 16, 465-468, 1971 :

Carcaterra, C., Il problema della fallacia naturalistica. La derivazione del dover essere dall'essere.

1971 Archives de Philosophie du Droit, 16, 489-494, 1971 :

Ferrajoli, L., Teoria assiomatizzata del diritto.

1981 Archives de Philosophie du Droit, 26, 419-423, 1981 :

Finnis, John, Natural Law and Natural Rights

1973 Revue philosophique et juridique de Louvain, 71, 1973 :

Gardies, J. G., Essai sur les fondements a priori de la rationalité morale et juridique.

1970 Revue philosophique de Louvain, 86, 407, 1970 :

\footnotetext{
${ }^{1}$ Ce choix parmi quelques centaines de recensions a été opéré par J. Kalinowski lui-même.
} 
Gauthier, D. P., Practical Reasoning.

1965 Archives de Philosophie du Droit, 10, 349-350, 1965 :

Goldschmidt, W., Der Aufbau der juristischen Welt

1973 Archives de Philosophie du Droit, 36, 321-328, 1973 :

Hilpinen, R. Deontic Logic : Introductory Readings

1970 Archives de Philosophie, 33, 161, 1970 :

Husserl, E., Briefe an Roman Ingarden.

1971 Archives de Philosophie du Droit, 16, 494-498, 1971.

Lampe, E. J., Juristische Semantik.

1969 Les études philosophiques, 24, 416-417, 1969 :

Perelman, Ch., Les lacunes en droit.

1968 Archives de Philosophie du Droit, 13, 421-422, 1968 :

Perelman, Ch., Les lacunes en droit.

1966 Archives de Philosophie du Droit, 11, 364-386, 1966 :

Perelman, Ch., Les Antinomies en droit.

1973 Archives de Philosophie, 36, 1973 :

Perelman, Ch., Le champ de l'argumentation.

1969 Archives de Philosophie du Droit, 14, 348-352, 1969.

Perelman, Ch., La théorie de l'argumentation.

1969 Archives de Philosophie du Droit, 14, 337-341, 1969.

Perelman, Ch., Droit, Morale et Philosophie

1969 Archives de Philosophie du Droit, 14, 399, 1969 :

Rescher, N., The Logic of Commands.

1969 Archives de Philosophie du Droit, 14, 397-399, 1969 :

Ross, A., Directives and Norms.

1959 Bulletin des Facultés Catholiques de Lyon, 73-77, 1959 :

Roure, M.-L., Logique et métalogique.

1968 Archives de Philosophie du Droit, 13, 374-375, 1968 :

Villey, M., Cours d'histoire de la philosophie du droit, fasc. V.

1965 Archives de Philosophie du Droit, 10, 355-361, 1965 :

Villey, M., Cours d'histoire de la philosophie du droit, fasc. I à V.

1963 Archives de Philosophie du Droit, 8, 298-301, 1963 :

Villey, M., Leçons d'histoire de la philosophie du droit. 
1970 Archiv für Rechts-und Sozialphilosophie, 56, 572-575, 1970 :

Wright, G. H. von, An Essay in Deontic Logic and the General Theory of Action.

1969 Archives de Philosophie du Droit, 14, 400-401, 1969 :

Wright, G. H. von, An Essay in Deontic Logic and the General Theory of Action.

1969 Archives de Philosophie du Droit, 14, 395, 1969 :

Wright, G. H. von, The Logic of Practical Discourse.

1964 Les études philosophiques, 19, 492, 1964 :

Wright, G. H. von, Norm and Action.

\section{- Principales traductions et éditions de textes de Jerzy Kalinowski}

\section{Martyniak, Czeslaw}

1949a Obiektywna podstawa prawa wedlug sw. Tomasza z Akwinu (tr. du fr. par Jerzy Kalinowski), Lublin : TNKUL, 1949.

1949b Problem filozofii prawa, jej przedmiot, metoda i podzial, Lublin (TNKUL), Jerzy Kalinowski (éd.), 1949.

\section{WEGRZECKI, ADAM}

1972 Roman Ingarden : l'homme et son œuvre, Les études philosophiques, 1, 55-60 1972.

TARski, A.

1972/74 Logique, sémantique, métamathématique, t. I, Paris : A. Colin, 157-269, 1972, et t. II, 1974, 203-242 et 265-305 (tr. fr. par Jerzy Kalinowski des études de Tarski : "Pojecie prawdy w jezykach nauk dedukcyjnych "; " The semantic conception of truth...";

"The completeness of elementary algebra and geometry").

\section{REgNeRI, CYPRIANI}

1986 Demonstratio Logicae verae iuridica, Georges Kalinowski (éd.), Bologna (C.L.U.E.B.), 1986. 
LeSNiEWski, StanislaW

1989 Sur les fondements de la mathématique. Fragments (Discussions préalables méréologie, ontologie), tr. du polonais par Georges Kalinowski, Paris : Hermes, 1989.

\section{- Principales études sur l'œuvre de Jerzy Kalinowski}

Bozzi, Rodolfo

1984 La lógica deóntica di Georges Kalinowski, Napoli : Ed. Jovene, 1984.

1981 La fondazione metafisica del diritto in Georges Kalinowski, Napoli : Ed. Jovene, 1981.

Cabanillas, Renato Rabbi-Baldi

1990 El concepto de derecho en el realismo clásico a partir de las opiniones de Michel Villey y Georges Kalinowski, Persona y Derecho. Revista de Fundamentación de las Instituciones Jurídicas y de derechos Humanos, 25, 73-114, 1990.

Hernandez, Manuel Ballester

1992 La Unidad del Pensamiento. Estudio sobre el itinerario intelectual de G. Kalinowski, Barcelona : PPU, 1992.

Herrera, Daniel Alejandro

2000 La noción de derecho en Villey y Kalinowski, Buenos Aires : EDUCA, 2000.

Massini, Carlos J.

1987 Derecho y Ley según Georges Kalinowski, Mendoza : Editorial Idearium, 1987.

WeinBerger, OtA

1960 Théorie des propositions normatives. Quelques remarques au sujet de l'interprétation normative des systèmes K1 et K2, Studia Lógica, 9, 7-21, 1960.

Voir aussi : http ://www.unipv.it/deontica/bibliog/b_d.htlm 1999 Dernière mise à jour : mai 1999. 